Mediterránea Ser. Biol. (1991), n. ${ }^{\circ}$ 13. Pág. 67-90

\title{
CARACTERIZACIÓN Y DISTRIBUCIÓN DE LA HERPETOFAUNA EN LA COMARCA DE ALICANTE SOBRE LA BASE DE SU MEDIO FÍSICO*
}

\author{
SPATIAL PATTERN OF A HERPETOLOGICAL COMMUNITY ACCORDING \\ TO ITS ENVIRONMENTAL VARIABLES IN ALICANTE REGION
}

\author{
por \\ LINA GRACIA, EDUARDO SEVA ${ }^{1}$ y CARLOS ORTIZ
}

\section{RESUMEN}

En la provincia de Alicante (S.E. España) se cuenta ya con ciertos estudios herpetológicos, observándose en todos ellos que existen especies ubiquistas y especies ligadas a determinadas condiciones ambientales.

El presente trabajo ha sido desarrollado en el territorio de la Comarca de L'Alacantí (536 $\mathrm{km}^{2}$ ) y tiene como objeto determinar la composición, características y patrones de distribución de la comunidad de Lacértidos que se asienta en el típico paisaje mediterráneo ampliamente sometido a la influencia humana.

Se ha efectuado un muestreo estratificado sobre 33 cuadrículas cuadradas de $250 \mathrm{~m}$. de lado, extraídas de una base de datos multitemáticos del medio físico de la comarca, incluyendo, por el método de selección, un total de 10 tipos distintos de biotopos (6 corresponden a vegetación natural y 4 a estructuras antropógenas). En cada uno de ellos se ha resgistrado la densidad de lacértidos. Para determinar la importancia que cada biotopo tiene sobre esta comunidad, se ha calculado la riqueza y las preferencias de habitat para cada especie, mediante análisis de Componentes Principales. En todo momento se describe la importancia das como la realización de programas de que tiene para cada especie la conservación de la estructura de vegetación natural.

PALABRAS ClaVE: Comunidad de Lacértidos, patrón de distribución, preferencias de habitat, Alicante, S.E. España.

\footnotetext{
* Trabajo subvencionado a través de una ayuda a la investigación al Instituto Gil-Albert. Diputación de Alicante. Convocatoria 1990.

1 Dpto. Ecología. Fac. Ciencias. Universidad Alicante. Apdo. 99. 03080 Alicante.
} 


\section{SUMMARY}

There are already some studies about reptiles in Alicante's province. In all of them, we can observe there are two different groups: ubiquitious ones and some linked to espatial environment conditions.

This present work is related to the region "L'Alacantî" (Alicante province, S.E. Spain) and its goal is to determine the composition, caracteristics and distribution patterns in a Lacertid community developed in its tipical mediterranean landscape, under human influence.

A stratified sampling method has been carried out over 33 square plots of $250 \mathrm{~m}$. side, take from an environment database of this region, including a total of 10 different biotopes ( 6 bellonging to the natural vegetation and 4 to those of humen influence), measuring abundance and density of them. To determine the importance of each biotope over this community, the health they present and the preferences of habitat have been calculated by means of a C.P.A. the vegetation type is important for each species along the whole work how is described aswell.

KEY WORDS: Lacertid community, distribution pattern, habitat preferences, Alicante, S.E. Spain.

\section{INTRODUCCIÓN}

En nuestra provincia contamos ya con algunos estudios herpetológicos (ESCARRE y VERICAD, 1981; SEVA, 1982; SEVA y ESCARRE, 1983). En todos ellos, se observa que las distintas especies se distribuyen en relación a una serie de características físicas o medioambientales. El medio ambiente influirá, por tanto, en la distribución geográfica y número de individuos de cada especie, dado que la riqueza de réptiles está condicionada por la complejidad estructural y diversidad de la vegetación (PÈREZMELLADO, 1983; SANTOS y TELLERIA, 1989).

En la comunidad herpetológica de la comarca de Alicante, podemos encontrar especies ubiquistas, que colonizan casi cualquier tipo de habitat, y especies que están ligadas a unas determinadas condiciones medioambientales; por tanto, se puede relacionar la diversidad faunística con el estado de conservación de cada uno de los biotopos, naturales y antropizados, que componen la comarca.

Es en esta línea, en la que el presente trabajo tiene como objeto determinar la composición y características de la comunidad herpetológica que se desarrolla en un típico paisaje mediterráneo con grandes áreas de influencia humana. Con ello se pretende conocer el estado de la comunidad, y la respuesta de cada especie a las modificaciones de habitat producidas por la actividad agrícola y urbanística, todo ello en un intento de utilizar a los réptiles como bioindicadores para elaborar posibles estrategias conservacionistas.

Para el desarrollo de este trabajo, se ha utilizado una amplia base de datos medioambientales de la zona, elaborada por los antiguos Departamentos de Geología, Biología y Bioestadística de la Universidad de Alicante, en 1981. 


\section{DESCRIPCIÓN DEL ÁREA DE ESTUDIO}

\subsection{Situación y extensión}

El área de estudio se sitúa en la comarca de L'Alacantí, exceptuando los términos de Jijona y Torremanzanas, y la isla de Tabarca, y añadiendo las partidas de Torrellano y El Altet pertenecientes al término municipal de Elche. Así pues, se han estudiado también los términos municipales de Alicante, San Vicente del Raspeig, Campello, San Juan, Aguas, Busot y Agost, con un total de $531 \mathrm{~km}^{2}$.

\subsection{Geografía física}

La comarca está incluida en la Zona Prebética, dentro del contexto de las Sierras Béticas. Presenta facies neríticas, y se divide en dos dominios: uno situado al norte denominado Prebético Externo, caracterizado por la presencia de materiales detríticos y dolomíticos, y otro situado al sur, denominado Prebético Interno, que posee series más potentes de calizas y margas (AUERHEIMER y PINA, 1983).

En relación con la geomorfología, se observa un claro contraste entre las Ilanuras aluviales (campos de San Juan y Muchamiel) y los glacis (de San Vicente principalmente), ambos de origen Cuaternario, y las montañas y sierras de origen Terciario (Jurásico y Cretácico) que en dirección ENE - WSW forman, al norte de la comarca, la alineación Bética.

En la costa existen zonas de acantilado y costa llana, con sedimentos en playas tendidas de arena, ocasionalmente grava, con los correspondientes colchones de dunas litorales, tan característicos en el sur (AUERNHEIMER et al., 1981).

\subsection{Climatología}

El área de estudio se encuentra dentro del dominio del Clima Mediterráneo, que está caracterizado por la existencia de un período xérico estival, donde aumenta fuertemente la evapotranspiración debido a la elevada temperatura, disminuyendo considerablemente las precipitaciones, siendo en ocasiones ausentes. Para cuantificar esta xericidad estival, se utiliza el Índice de Mediterraneidad, siendo para Alicante igual a 17.3 el más bajo del sureste peninsular, y por tanto la zona menos xérica de este cuadrante (Murcia: 31.9 y Almería: 58.7, según RIVAS-MARTÍNEZ, 1987).

La Temperatura media anual $(\mathrm{Tm})$, en Alicante es de $17.4^{\circ} \mathrm{C}$ (entre 1968 y 1983), observándose temperaturas bastante suaves, cuyos valores medios máximos se alcanzan en agosto con $25.1^{\circ} \mathrm{C}$, y los mínimos en enero con $11.5^{\circ} \mathrm{C}$, con un intervalo de variación anual de $13.6^{\circ} \mathrm{C}$.

La entrada del verano se produce en junio, con un aumento de $4^{\circ} \mathrm{C}$, y la entrada del otoño en octubre con un descenso de $4.1^{\circ} \mathrm{C}$. La temperatura media no desciende nunca de $11.5^{\circ} \mathrm{C}$, y pasa de los $20^{\circ} \mathrm{C}$ los meses 


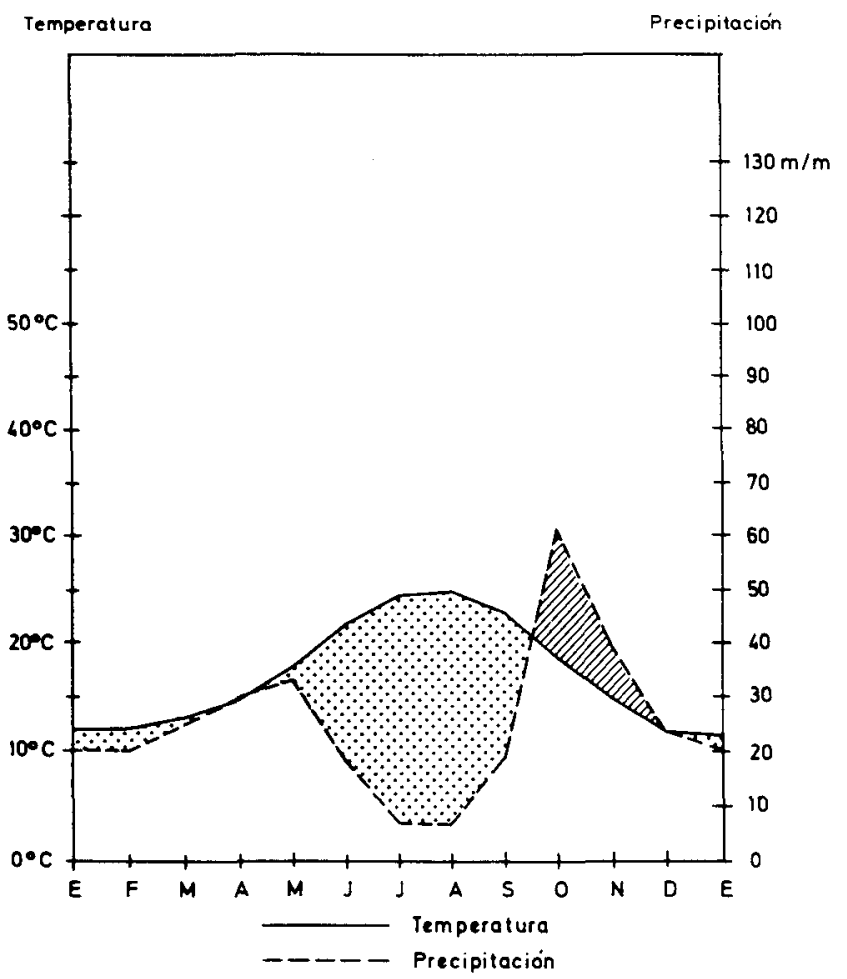

Figura 1.- Diagrama ombrotérmico de Gaussen.

de junio, julio, agosto y septiembre, lo que indica, según el termograma de Jefferson, que la mayor parte del año ( 8 meses) se considera estación primaveral (enero a mayo), estableciéndose tan sólo dos estaciones más: verano (de junio a septiembre) y otoño (de octubre a diciembre) (BARTOLOMÉ, 1985).

En relación con la precipitación, a lo largo del período considerado, se observa una distribución irregular con valores que van desde $172.6 \mathrm{~mm}$. de Precipitación media anual (Pm) en 1970, hasta $531.1 \mathrm{~mm}$. enn 1982 . La Pm para todo el período considerado es de $302.4 \mathrm{~mm}$., con el máximo en octubre $(61.2 \mathrm{~mm})$ y el mínimo en agosto $(6.7 \mathrm{~mm}$ ) (BARTOLOMÉ 1985$)$.

Si observamos el diagrama ombrotérmico de Gaussen (Figura 1), podemos ver que el período seco abarca bastantes meses del período anual.

Según estos datos, se encuadra la comarca de Alicante dentro del dominio del piso bioclimático Termomediterráneo $\left(17^{\circ} \mathrm{C}<\mathrm{Tm}<19^{\circ} \mathrm{C}\right)$, con un ombroclima Semiárido $(200 \mathrm{~mm}<\mathrm{Pm}<350 \mathrm{~mm})$, según el criterio de RIVAS-MARTÍNEZ, 1987. No obstante, se observa una enorme variabilidad en los valores medios con diferencias notables de unos años a otros (C.V. $30 \%$ ) y por ello el ombroclima varía entre Semiárido y Seco (350 $\mathrm{mm}<\mathrm{Pm}<600 \mathrm{~mm}$ ). 


\subsection{Vegetación}

El área de estudio se corresponde con el Sector Alicantino, dentro de la provincia corológica Murciano-Almeriense (ALCARÁZ y PEINADO, 1987).

En relación con la cubierta vegetal que se encuentra la comarca de L'Alacantí, se han definido 11 tipos distintos de vegetación (ESCARRE et al., 1981; ESCARRE et al., 1983; RIGUAL, 1972).

1.- Carrascal: Se trata de un bosque esclerófilo de Quercus ilex ssp rotundifolia muy pobre en especies. Fitosociológicamente, se encuentra dentro de la asociación Quercetum-rotundifoliae, y tan sólo se presenta de forma puntual, localizado en el Cabeço d'Or y en la umbría de la Sierra del Ventós.

2.- Maquia de espino, coscoja y palmito: Pertenece a la clase Quercetea-ilicis, dentro de la alianza Oleo-Ceratonion, representada por dos asociaciones: Querco-lentiscetum y Chamaeropo-Rhamnetum. La primera es una maquia densa de coscoja y lentisco ( $Q$. coccifera y Pistacia lentiscus), y la segunda es un espinar aclarado con palmito, espárragos y uvas de mar (Chamaerops humilis, Asparagus stipularis y A. albus y Ephedra fragilis). En ningún caso se obtiene un tapiz continuo, sino que se trata de retazos de maquia combinados frecuentemente con romeral.

3.- Romeral: Se encuentra dentro de las clase Ononido-Rosmarinetea en el orden Rosmarinetalia y la alianza Rosmarino-Ericion. Se trata de un matorral formado por romero, lino y esparto (Fumano-Stipetum tenacissimae), a veces con dominio del esparto sobre las otras especies, o por romero combinado con jara blanca (Anthyllido-cistetum clussi). En gran parte de este biotopo se observa una transición gradual hacia el tomillar.

4.- Tomillar: Se encuentra en la misma clase que el romeral, pero representado por el orden Anthyllidetalia, con la alianza Thymo-Siderition. Es el biotopo más extenso de la comarca, y el que mejor representa las condiciones de aridez que se dan en este territorio, con la particularidad de estar formado por un grupo de especies endémicas de mediterráneo meridional e iberomauritánicas.

5.- Vegetación gipsófila: Se encuentra en los sustratos yesíferos, que presentan suelos esqueléticos en los que aflora el yeso; sobre ellos se desarrolla una cubierta vegetal muy escasa. En la taxonomía fitosociológica se encuentran dentro de la clase Ononido-Rosmarinetea, al igual que los romerales y tomillares, no siendo muy diferente de estos últimos, pero constituido por el orden Gypsophiletalia, y representado por la alianza Gypsophilion. En esta comunidad vegetal suelen aparecer especies que también son características del tomillar.

6.- Pradera de gramíneas: se corresponde, sobre todo, con la alianza Hyparrhenion-hirtae, y constituye lastonares de zonas muy degradadas. Está escasamente representada, ocupando tan sólo un $0.7 \%$ de la extensión de la comarca.

7.- Vegetación de baladre y murta: Se encuentra representada por la alianza Nerion-oleandri, que es el conjunto de comunidades propias de las 
ramblas formadas por los ríos de la región mediterránea meridional, con inundaciones esporádicas. Está formado, casi exclusivamente, por baladre (Nerium oleander), y tan solo en los puntos más resguardados aparecen también taray y murta (Tamarix sp. y Mirtus communis).

8.- Vegetación psammófila: Se corresponde con la clase Ammophiletea, en el orden Ammophiletalia, representado por tres alianzas zonificadas según su proximidad al mar. Es la cubierta vegetal propia de zonas arenosas costeras, y se encuentra tan sólo en la playa del Saladar y Cabo de la Huertas, con una fuerte acción antrópica.

9.- Vegetación halófila: Esta unidad se encuentra en la clase Puccinellio-Salicornietea, que engloba a las comunidades vegetales propias de suelos arcillosos y con altas concentraciones salinas. Son comunidades fruticosas o herbáceas, generalmente con pocas especies. Para los Balsares de El Altet, que es el área más extensa con este tipo de vegetación, se han descrito las asociaciones Salicornio-Suaedetum brevifoliae y Arthrocnemetumfruticosi.

10.- Vegetación de terrenos improductivos: Esta es sin duda la unidad vegetal más heterogénea, ya que comprende toda la serie progresiva de comunidades que se establece sobre un campo cultivado en el momento que cesa su explotación, y por lo tanto la colonización se realiza en circunstancias muy distintas según la situación de éste respecto a la vegetación natural de la zona. La vegetación que cubre estos campos abarca varias unidades taxonómicas, la clase Rudero-Secalietea, especialmente las alianzas Diplotaxion y Salsolo-Peganion, y la clase Thero-Brachypodietea representada por praderas de la alianza Stipion-retortae.

11.- Cultivos: Con relación a éstos, cabe señalar la existencia del almendro como cultivo mayoritario. Es de destacar dos tipos de cultivos en regresión, el almendero y el olivo, y dos en expansión, el tomate y la vid. También tienen una considerable representación dentro de este tipo de vegetación, las zonas ajardinadas. Dentro de los cultivos se han considerado también las repoblaciones de eucaliptos y pinos, la primera muy poco extendida, y la segunda destacan las repoblaciones jóvenes, densas pero bastante escasas.

\section{MATERIAL Y MÉTODOS}

Del total de cuadrículas de $250 \mathrm{~m}^{2}$ en que se dividió la comarca para el estudio del medio físico (AUERNHEIMER y ESCARRE, 1981), se ha obtenido una muestra de 33, distribuidas al azar por toda la zona de estudio, consideradas como las más idóneas dentro de una escala de representatividad del total de cuadrículas.

Para ello, se procedió a ordenar el conjunto de cuadrículas, aceptando la hipótesis de proporcionalidad de la densidad poblacional con la superficie de vegetación natural que contiene cada una de ellas, y distribución al azar de los individuos, lo que da lugar a una dịstribución de Poisson en los mues- 
treos con un parámetro $\lambda$ y desviación típica $\lambda^{1 / 2}$. El procedimiento es el siguiente:

1. Se asigna a cada cuadrícula una medida igual a la raíz cuadrada del porcentaje de vegetación natural en ella.

2. Se le asigna como probabilidad su medida particular normalizada para el conjunto de la comarca y se ordena finalmente en el intervalo [0, 1].

3. Para la obtención de la muestra se generan $n$ números aleatorios en este intervalo, y se identifican las $n$ cuadrículas que los contengan.

Para realizar la prospección en ellas, se ha establecido un muestreo estratificado, que consiste en delimitar dentro del área elegida una serie de unidades de muestreo más homogéneas, en función de diferentes unidades fitosociológicas de vegetación (SCHERRER, 1983), que denominaremos biotopos.

Este tipo de muestreo presenta una serie de ventajas enunciadas por CAUGHLEY, (1977): a) disminuye la dispersión de los resultados al disminuir la variabilidad de la densidad en el área seleccionada para la prospección, b) proporciona una eficacia y dosificación del esfuerzo al permitir aumentarlo o disminuirlo dependiendo de la abundancia del biotopo, c) las densidades calculadas para cada biotopo pueden ser comparadas entre sí, aportando una información biológica interesante.

En relación con estos estratos de muestreo, hemos considerado un total de diez biotopos distintos, divididos en vegetación natural, terrenos improductivos, terrenos de cultivos y repoblaciones, y estructuras antropógenas.

Dentro de la vegetación natural de la zona, se hallan representados 6 biotopos, de los que faltan el carrascal y la pradera de gramíneas, que por su escasa representación en la comarca se encuentran fuera de las cuadrículas elegidas para el muestreo, y la vegetación gipsófila, que por su fisonomía y composición vegetal tan próximas al tomillar (RIGUAL, 1972), se ha incluido en este último biotopo, ya que para la fauna no se han considerado las diferencias edáficas en una u otra vegetación. Así se ha clasificado la vegetación natural en: maquia de espino, coscoja y palmito (MAQ), romeral (ROM), tomillar (TOM), vegetación de baladre y murta de ramblas (RAM), vegetación psammófila de arenas de playas y dunas (APD), y vegetación halófila de saladares (SAL).

Los terrenos improductivos (IMP), y los cultivos y repoblaciones (CUL), han sido considerados tal y como se describen en el apartado 2.4.

Las estructuras antropógenas han sido consideradas como dos biotopos distintos, uno correspondiente al antropógeno urbano (URB), donde se encuentran viviendas urbanas, urbanizaciones intensivas, autopistas y carreteras asfaltadas, y otro como antropógeno rústico (RUS), donde se hallan las casas de campo, construcciones aisladas, ruinas puntuales, balsas, acequias y caminos.

Para desarrollar el muestreo es importante conocer bien el momento del ciclo estacional en que se realiza, con el fin de interpretar adecuada- 


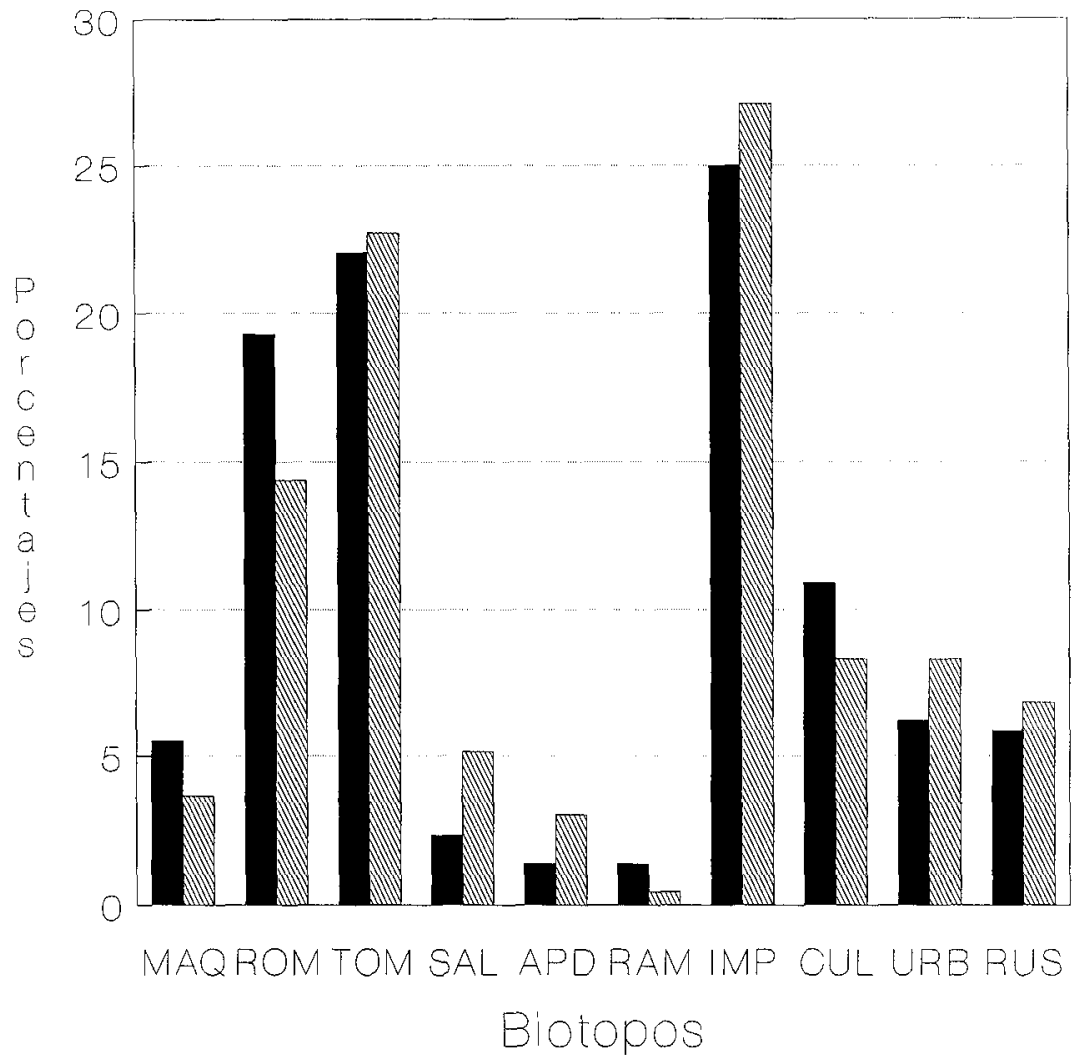

PROSPECCION MW SUPERFICIE

Figura 2.- Comparación de las distribuciones de los porcentajes de muestreo y superficie, en relación con los biotopos prospectados en la comarca $\left(\mathrm{X}^{2}=8.67, \mathrm{p}>0.05\right)$.

mente los resultados obtenidos, y poder llevar a cabo comparaciones en la población muestreada en momentos equivalentes de su ciclo anual de abundancia (PÉREZ-MELLADO, 1989).

Para ello se han establecido cuatro rondas de muestreo correspondientes a las estaciones en las que se encuentran más activos los réptiles: primavera, verano y otoño. La prospección comenzó en primavera de 1989 y finalizó en primavera de 1990.

En cada estación se visitaron las cuadrículas en las horas de máxima actividad para cada período, estableciéndose una serie de transectos lineales a pie por cada biotopo. Este método de contacto visual, parte del hecho de que la distribución está determinada por un proceso de Poisson no-homogéneo, y exige mantener constante el esfuerzo aplicado en cada unidad, por lo que se ha controlado la longitud y velocidad de progresión (TELLERÍA, 1986). 
Se ha considerado como unidad de muestreo $100 \mathrm{~m}$. lineales por $5 \mathrm{~m}$ de amplitud visual $\left(500 \mathrm{~m}^{2}\right)$, con una duración aproximada de 5 minutos, mediante los que se han prospectado un total de $145.000 \mathrm{~m}^{2}$, equivalentes a 290 unidades de muestreo, y se han levantado del orden de 3.000 piedras.

La distribución del porcentaje de prospección en cada biotopo es representativa de la presencia de éstos en el área de estudio $\left(\mathrm{X}^{2}=8.67 \mathrm{p}>\right.$ 0.05) (Figura 2.).

Para el cálculo de la abundancia hemos utilizado el índice descrito por LIZANA et al. (1987), definido por la expresión:

$$
\mathrm{I}=\left(\mathrm{n}_{\mathrm{i}} / \mathrm{N}_{\mathrm{i}}\right) / \sum\left(\mathrm{n}_{\mathrm{i}} / \mathrm{N}_{\mathrm{i}}\right)
$$

siendo $n_{i}$ el número de individuos encontrados en el recurso $i$, y $N_{i}$ el número de unidades de muestreo realizadas en el mismo recurso. El resultado lo hemos multiplicado por cien para expresarlo en porcentajes.

Para medir el grado de solapamiento hemos utilizado la expresión:

$$
S=\left(\sum P_{i j} \cdot P_{i k}\right) /\left(\sum P_{i j} \cdot \sum P_{i k}\right)^{1 / 2}
$$

propuesta por MACARTHUR y LEVINS (1967) y simetrizada por MAY (17974), donde $\mathrm{P}_{\mathrm{ij}} \mathrm{P}_{\mathrm{ik}}$ son las proporciones respectivas que presentan las especies j y k en el recurso $i$.

Los dendrogramas de afinidad han sido construidos mediante el método de Análisis de Agrupamiento ("Cluster Analysis"). Para ello se ha utilizado, como criterio de agrupamiento, el promedio de grupo.

\section{RESULTADOS Y DISCUSIÓN}

Se ha obtenido un total de 262 citas, de las que 186 corresponden a datos obtenidos en la prospección de campo. El resto se ha extraido mediante la revisión del archivo faunístico del Departamento de Ciencias Ambientales y Recursos Naturales de la Universidad de Alicante, anotando las citas de réptiles cuya localización coincidía con las cuadrículas objeto de estudio. No obstante, estas últimas citas no pueden ser cuantificadas en relación con los esfuerzos de prospeción, por lo que no se han utilizado en los análisis estadísticos correspondientes.

De las 15 especies descritas para la comarca de Alicante (ESCARRE y VERICAD, 1981) han sido detectadas 13 mediante prospección, coincidiendo con las mismas especies que se encontraban descritas en los datos de archivo para las cuadrículas objeto de estudio. Las especies encontradas se distribuyen en cuatro familias:

FAMILIA AMPHISBAENIDAE:

Blanus cinereus

FAMILIA GEKKONIDAE:

Hemidactytus turcicus

Tarentola mauritanica 
FAMILIA LACERTIDAE:

Acanthodactylus erythurus

Lacerta lepida

Podarcis hispanica

Psammodromus algirus

Psammodromus hispanicus

FAMILIA COLUBRIDAE:

Coluber hippocrepis

Coronella girondica

Elaphe scalaris

Malpolon monspessulanus

Natrix maura

Las especies que no han sido localizadas en los muestreos son: Chalcides bedriagai y Macroprotodon cucullatus. La primera tan sólo muestra una alta densidad dentro de nuestra comarca en lá Isla de Tabarca (SEVA y ESCARRE, 1976), localidad que queda fuera del área objeto de estudio, la segunda pertenece al grupo de los Oficios, que en general son huidizos y difíciles de detectar. A esta dificultad se suma la característica de ser la especie menos abundante de la comarca (VERICAD y ESCARRE, 1981) y presentar hábitos nocturnos (SALVADOR, 1985), lo que dificulta aún más su detectabilidad.

El mayor número de citas se obtiene con $P$. hispanicus, seguido a distancia por P. hispanica y L. lepida (Figura 3). En general las citas de los reptiles no Saurios son escasas, tal y como ocurre en otros estudios de comunidades similares (LIZANA et al., 1987), características que es importante destacar, ya que, dado el gran número de prospecciones realizado bajo piedras, pensamos que estos resultados responden realmente con una menor densidad de Ofidios. Aceptando la hipótesis de proporcionalidad entre los muestreos y la obtención de citas, tendríamos que multiplicar, como mínimo por diez, el número de transectos para contar con unos resultados similares a los obtenidos para los Saurios.

Cabe destacar también la baja abundancia poblacional del área de estudio, estimada entre 12 y 13 individuos por hectárea muestreada, que si bien es algo superior a la encontrada por SANTOS y TELLERIA (1989) para Lacértidos en medios cerealistas del centro de España (entre y 7 ind.I ha muestreada), se debe probablemente a que ésta última se trata de una zona con menor diversidad ambiental que la de nuestra comarca. Sin embargo, ambos valores están en contraste con los obtenidos en biotopos mejor conservados (unas 10 veces superiores, CANO, 1984).

Para obtener una primera representación de la estructura de la comunidad se ha construido un dendograma (Fig. 4), en base a las abundancias específicas encontradas en cada cuadrícula, teniendo en cuenta el valor de las variables medioambientales consideradas en cada una de ellas (altitud, orientación, pendiente y porcentaje de ocupación del biotopo, y diversidad, altura y cobertura de la vegetación). 


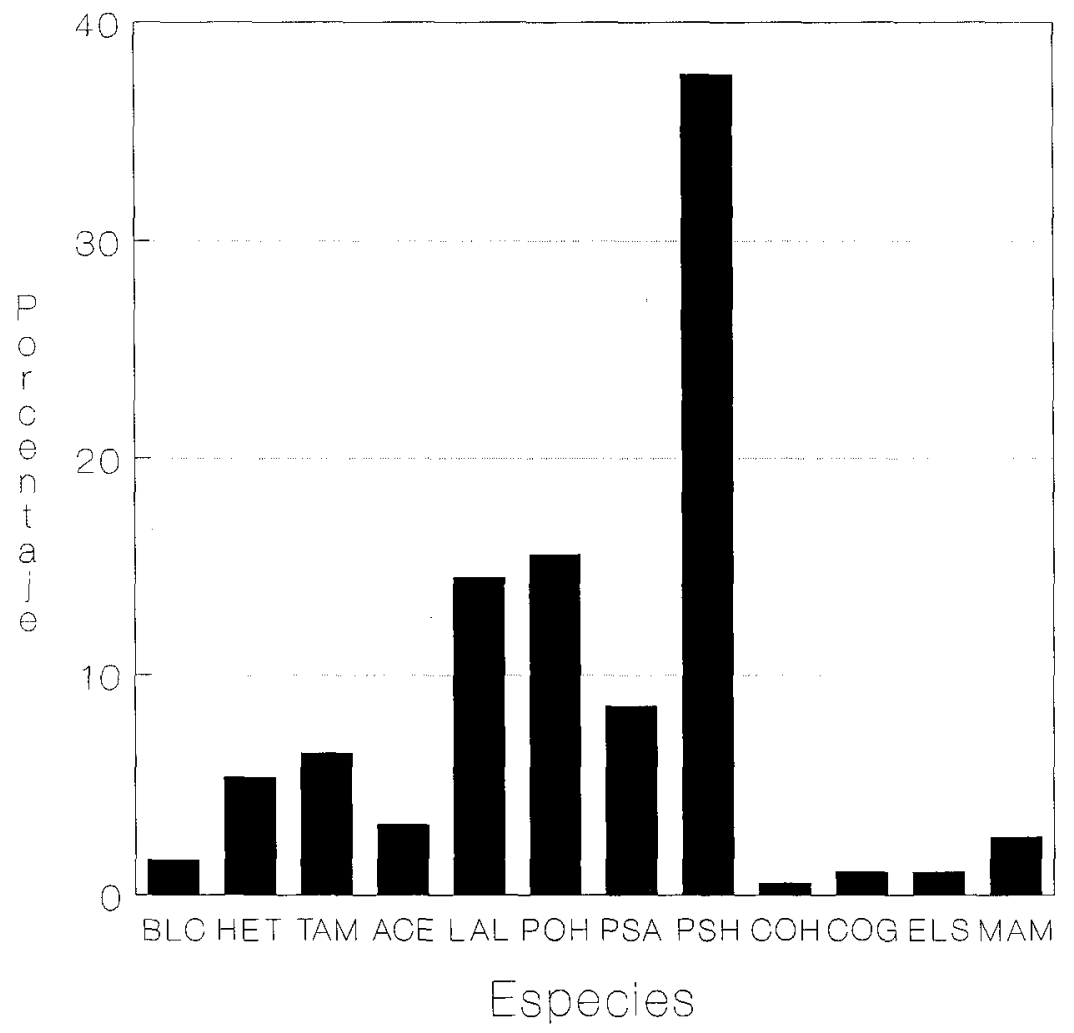

Figura 3.- Porcentaje de contactos de cada especie.

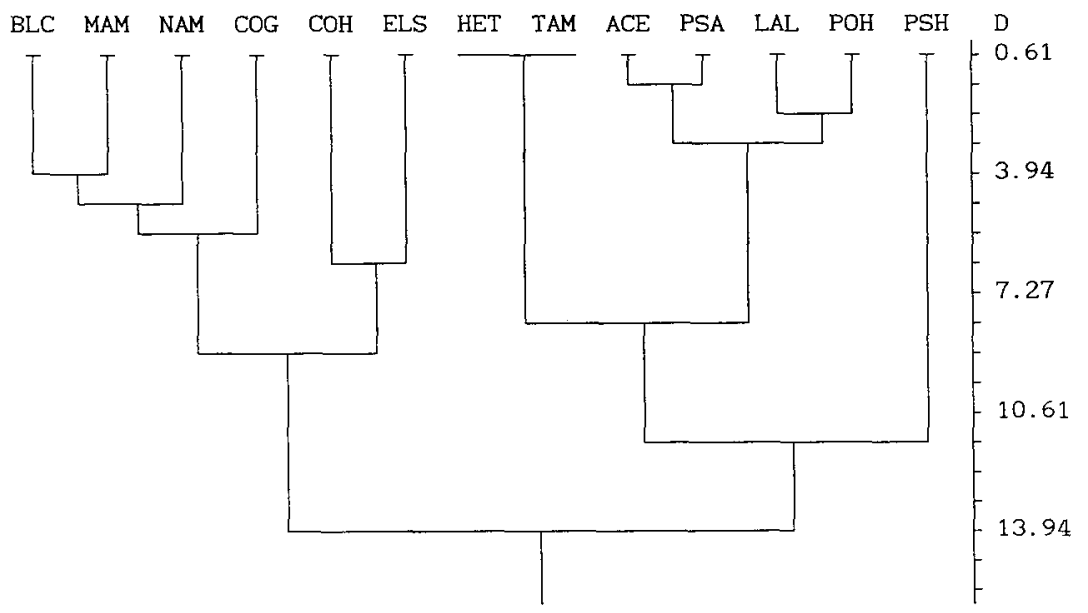

Figura 4.- Dendrograma de Afinidad obtenido mediante Análisis de Agrupamiento de la comunidad de reptiles en relación con las cuadrículas prospectadas. 
Se observan dos grandes grupos, uno de Saurios y otro de Ofidios y Anfisbaénidos, tal y como cabría esperar con sus costumbres y detectabilidad. En el primer grupo existen asociaciones entre especies de hábitos y exigencias similares (ARNOLD y BURTON, 1978; ESCARRE y VERICAD, 1981), aunque existe una clara separación por parte de $P$. hispanicus con el resto de los Saurios, debido a que ha sido el más abundante y menos exigente en cuanto a requerimientos ambientales. En relación con el segundo grupo, existe una asociación clara entre los Ofidios, como era de esperar, aunque también se incluye la especie $B$. cinereus de otro taxon, debido a que este grupo basa su asociación, principalmente, en la característica común de baja densidad frente a los Saurios.

\subsection{Preferencias de habitat}

Para resaltar los patrones en la ordenación de la comunidad y la búsqueda de similitudes, se ha efectuado en Análisis de Componentes Principales, en el que se han determinado las coordenadas de las especies en el espacio definido por los diez biotopos. Se acepta la suposición implícita de linearidad de estas coordenadas respecto a las abundancias en cada biotopo, y se toman para el análisis los tres primeros ejes.

En la Tabla I se puede observar el peso que cada biotopo tiene en los tres primeros vectores propios, asociados a los tres primeros ejes, y las coordenadas de las especies para cada uno de ellos.

Limitaremos los comentarios a los Saurios, debido a que son los que tienen mayor representación y densidad suficiente para el análisis estadístico.

El primer eje está fuertemente correlacionado con los terrenos improductivos, y la especie con mayor coordenadas sobre él es PS. hispanicus, seguido a distancia por $L$. lepida.

El segundo eje representa principalmente a tres biotopos de la vegetación natural: romeral, tomillar y maquia. Ya que sus valores se obtienen en sentido negativo, las máximas coordenadas asociadas han de ser negativas, y vienen dadas para $P$. hispanica, $L$. lepida y $P$ s. algirus, que prodríamos considerar las más ligadas a estos medios y más representativas de la vegetación natural actualmente dominante en la comarca, ya que las zonas de maquia, aunque puntuales por sí solas, se pueden encontrar frecuentemente asociadas al romeral.

Se observa que es $L$. lepida la especie más ecléctica, colonizando fácilmente la vegetación natural y los terrenos improductivos.

La interpretación del tercer eje no es tan clara numéricamente, ya que hay menos diferencias en las cargas de los diferentes biotopos, pero se aprecia un peso importante, en el sentido positivo, en las zonas de arenal, y en el sentido negativo, en los terrenos antropizados urbanos y rústicos. Las especies con mayores valores positivos son $A$. erythurus yPs. algirus, y por tanto las mejores colonizadoras de los medios dunares. Esta tendencia se manifiesta preferentemente en $A$. erythrurus que, debido a que su morfolo- 
TABLA I

VECTORES PROPIOS

\begin{tabular}{lccc}
\hline BIOTOPOS & VECTOR 1 & VECTOR 2 & VECTOR 3 \\
\hline MAQ & 0.0102 & -0.5134 & 0.2681 \\
ROM & 0.1088 & -0.5304 & 0.3523 \\
TOM & 0.0633 & -0.6243 & -0.2948 \\
SAL & 0.0374 & 0.0013 & 0.0503 \\
APD & -0.0186 & 0.0929 & 0.5139 \\
RAM & 0.0010 & -0.0784 & 0.0226 \\
IMP & 0.9858 & 0.0998 & -0.0330 \\
CUL & 0.0979 & 0.0006 & 0.0632 \\
URB & 0.0084 & 0.0183 & -0.4118 \\
RUS & -0.0287 & -0.2009 & -0.5247 \\
\hline
\end{tabular}

COORDENADAS DE LAS ESPECIES

\begin{tabular}{lccc}
\hline ESPECIES & EJE $\mathbf{1}$ & EJE 2 & EJE 3 \\
\hline BLC & 1.05 & -0.10 & -0.49 \\
HET & 0.10 & -2.62 & -4.22 \\
TAM & 2.20 & -2.22 & -3.70 \\
ACE & -0.11 & 0.56 & 3.08 \\
LAL & 1.87 & -5.65 & 1.15 \\
POH & 1.35 & -14.43 & -0.68 \\
PSA & 2.77 & -5.06 & 3.05 \\
PSH & 50.84 & -1.81 & -0.74 \\
COH & 0.06 & -0.62 & -0.29 \\
COG & 0.13 & -1.25 & -0.59 \\
ELS & 0.11 & -0.51 & 0.33 \\
MAM & 0.00 & -1.45 & -0.56 \\
NAM & 0.10 & -1.45 & -1.11 \\
\hline
\end{tabular}

Tabla I.- Resultados del Análisis de Componentes Principales para los tres primeros ejes.

gía está adaptada a los sustratos blandos (SEVA, 1982), su habitat se encuentra bastante restringido a los sistemas dunares (MELLADO, 1980; SEVA y ESCARRE, 1983). En el otro sentido, los mayores valores negativos se recogen para $H$. turcicus y $T$. mauritanica, especies cuya presencia está ligada por tanto a las construcciones humanas.

\subsection{Abundancias poblacionales}

El resultado global de la abundancia encontrada está expresado en la Tabla II, donde se observan máximos en esta magnitud para arenales costeros y maquia, con densidades relativas de muestreo estimadas en 45 y 21 individuos por hectárea, respectivamente, y mínimas para cultivos, estructuras antropógenas urbanas y romeral, con densidades relativas entre 7 y 8 individuos por hectárea para estos tres últimos biotopos. 


\begin{tabular}{lcccccccccc}
\hline BIOTOPOS & MAQ & ROM & TOM & SAL & APD & RAM & IMP & CUL & URB & RUS \\
\hline IND. AB & 13.8 & 5.1 & 6.7 & 5.7 & 29.1 & 6.5 & 11.8 & 4.9 & 5.0 & 11.4 \\
\hline
\end{tabular}

Tabla II.- Índice de Abundancia de reptiles encontrados en cada biotopo.

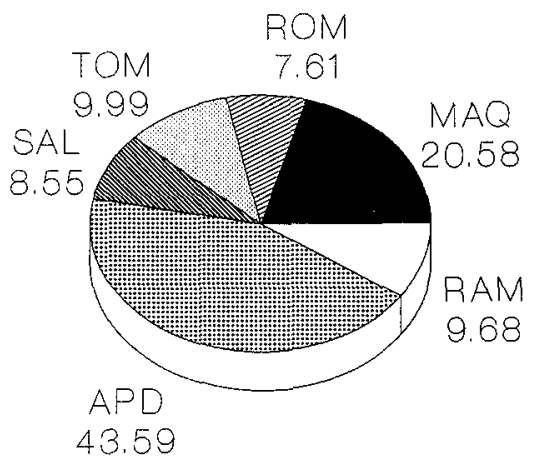

A

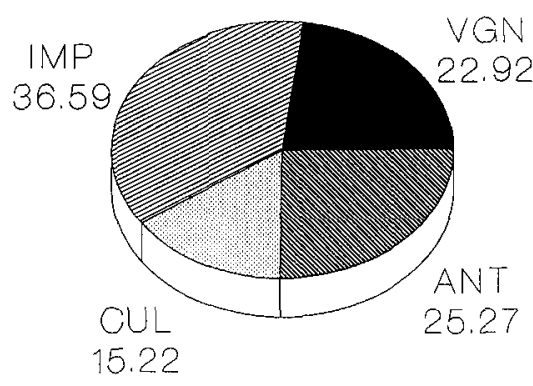

B

Figura 5.- Comparación del Índice de Abundancia de reptiles en los distintos biotopos; a) para la vegetación natural, b) para los biotopos agrupados.

Si agrupamos la vegetación natural (Figura 5a) podemos observar que siguen dominando los mismos biotopos, y continúa existiendo un mínimo en el romeral. Al establecer los biotopos en grandes unidades (Figura 5b), domina el improductivo sobre los demás, y existe un mínimo en los cultivos, ya que este último, presenta muchos cambios estructurales a lo largo del año, lo que hace muy difícil su colonización.

Estas abundancias máximas que se observan en las zonas de arenal y terrenos improductivos, se deben a la gran colonización que se observa por parte de algunas especies mejor adaptadas a estos biotopos, y no a una abundancia poblacional general. En el primer caso se debe, como ya hemos visto antes, a la colonización por parte de $A$. erythrurus de los arenales costeros alicantinos (ver ap. 4.1.; SEVA et al., 1982), y en el segundo caso se debe a una abundancia máxima para Ps. hispanicus, que se ve favorecida con la aparición de terrenos improductivos o baldíos (ver ap. 4.1.; SANTOS y TELLERÍA, 1989). 


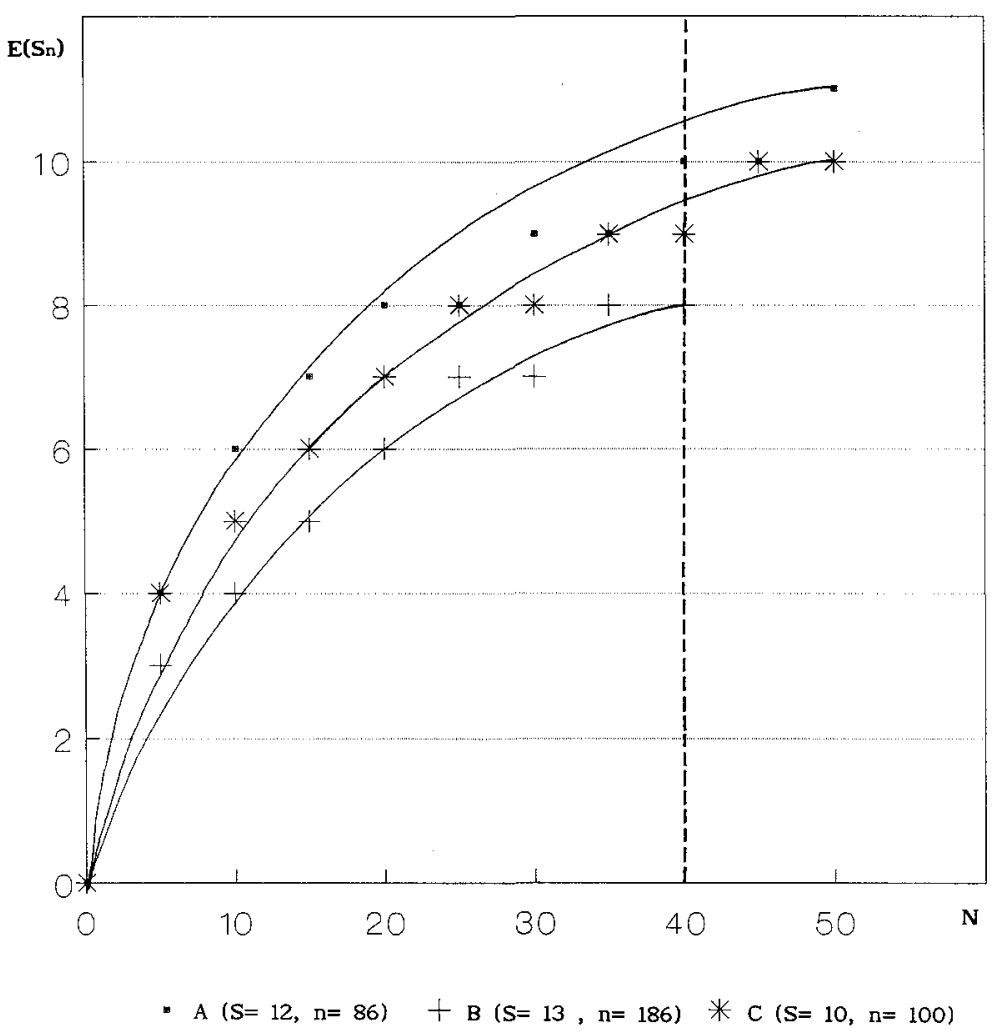

Figura 6.- Curva de Rarefacción entre el tamaño de muestra (N) y el $\mathrm{n} .{ }^{\circ}$ esperado de especies $\left(E\left(S_{n}\right)\right)$ en la vegetación natural $(A)$, en el total de los biotopos $(B)$, y en terrenos con mayor influencia humana $(C)$. $\left(S=n .{ }^{\circ}\right.$ de especies, $n=n .{ }^{\circ}$ de individuos.

\subsection{Diversidad y riqueza}

Para caracterizar la comunidad que se desarrolla en los distintos biotopos estudiados, hemos utilizado los índices de diversidad. En primer lugar hemos calculado diversidades generales mediante un proceso de rarefacción, que mide la riqueza potencial de un determinado recurso (JAMES y RATHBUN, 1981; MARGALEF, 1986). Este procedimiento estadístico es aplicable a muestras de tamaño diferente, y su objetivo es comparar el número de especies para un tamaño de muestra dado en las diferentes situaciones estudiadas, y suponiendo en todas ellas el mismo modelo de muestreo.

En nuestro caso hemos aplicado el procedimiento a la comunidad herpetológica estudiada en su conjunto, a los individuos detectados en medios naturales, y a los detectados en zonas de fuerte influencia antrópica, obteniendo las curvas representadas en la Figura 6. 
Se comprueba que los medios antropizados, cultivados y, en general, impactados por el hombre, muestran una menor riqueza de reptiles frente a la vegetación natual, que se muestra como el medio más rico, y por tanto mejor colonizado. Cabe destacar, que la riqueza de este último medio está ligeramente por encima de la curva patrón que se observa para latotalidad de biotopos descritos en la comarca, y por ello es de asumir la importancia que tiene, para la fauna en general, mantener una vegetación natural bien conservada.

Para detallar la importancia que cada biotopo tiene sobre la fauna herpetológica, hemos calculado el índice de diversidad de Shannon-Weaver (1957), y la equitabilidad de Pielou (1977), como medida del grado de organización de la comunidad (Tabla III).

Dentro de la vegetación natural, la máxima organización se encuentra en el tomillar, la maquia y el romeral. El primer caso constituye el biotopo de vegetación natural más extenso de la comarca (ESCARRE et al., 1983), y por lo tanto el menos fragmentado y más asequible para la herpetofauna. El romeral también se revela como biotopo importante, pero está más fragmentado (obs. per.), y en ocasiones, en gran parte de este biotopo se observa una transición gradual hacia el tomillar. La maquia constituye el medio más complejo y menos degradado de los que hemos considerado en nuestros muestreos, y pese a su gran fragmentación y esporádica y puntual aparición en la comarca, tiene gran importancia para el desarrollo de la fauna, en este caso concreto, para la comunidad herpetológica característica del sureste peninsular.

El resto de biotopos de vegetación natural, se presentan de forma tan puntual, que su estudio, dentro del conjunto de la comarca, se corresponde con un nivel de prospección muy pequeño para que no existan sesgos en favor de estos biotopos más escasos. Por ello, el muestreo no ha ofrecido los datos suficientes para destacar su importancia.

En el caso de biotopos influidos por el hombre, se observa una diversidad alta, efecto numérico debido más a la escasez de citas y aparición puntual de individuos, que a una representación real de la comunidad de reptiles. Sin embargo, en el medio antropógeno rústico, se encuentran valores de diversidad altos, frente al medio urbano, debido a que las edificacio-

TABLA III

\begin{tabular}{lrrrrrrrrrr}
\hline BIOTOPOS & MAQ & ROM & TOM & SAL & APD & RAM & IMP & CUL & URB & RUS \\
\hline $\mathrm{S}$ & 6 & 4 & 10 & 2 & 3 & 2 & 5 & 6 & 3 & 6 \\
$\mathrm{n}$ & 17 & 22 & 33 & 3 & 9 & 2 & 66 & 12 & 7 & 15 \\
$\mathrm{H}^{\prime}$ & 1.51 & 1.37 & 2.06 & 0.64 & 0.85 & 0.69 & 0.78 & 1.53 & 1.00 & 1.66 \\
$\mathrm{E}$ & 0.84 & 0.98 & 0.90 & 0.92 & 0.77 & 1.00 & 0.49 & 0.86 & 0.91 & 0.92 \\
\hline
\end{tabular}

Tabla III_- Diversidad de Shannon-Weaver (H') y equitabilidad de Pielou (E) de la comunidad de reptiles, para cada biotopo. ( $S=n^{\circ}$ de especies, $n=n^{\circ}$ de individuos). 


\begin{tabular}{lccc}
\hline RELACIÓN & $\mathbf{r}$ & $\mathbf{p}$ & $\mathbf{n}$ \\
\hline N.A. $=0.43 \times$ N.V. -2.87 & 0.866 & $<0.05$ & 7 \\
N.A. $=2.22 \times$ D.V. -0.90 & 0.820 & $<0.05$ & 7 \\
N.A. = A $\times$ A.V. + B & & N.S. & 7 \\
N.A. $=$ A $\times$ C.V. + B & & N.S. & 7 \\
H'A. $=0.077 \times$ N.V. -0.197 & 0.867 & $<0.05$ & 7 \\
H'A. = $0.438 \times$ D.V. +0.790 & 0.890 & $<0.01$ & 7 \\
H'A. = A $\times$ A.V.+B & & N.S. & 7 \\
H'A. = A $\times$ C.V. + B & & N.S. & 7 \\
\hline
\end{tabular}

Tabla IV.- Correlaciones y Regresiones lineales entre riqueza animal y altura (A.V.), grado de cobertura (C.V.) y riqueza vegetal en los biotopos naturales y terrenos improductivos (N.A., N.V. $=\mathrm{n}^{\circ}$ de especies animales y vegetales;H'A., D.V.= Diversidad animal y vegetal).

nes rústicas y puntuales, y otras construcciones dispersas que no afecten, en gran medida, a la vegetación circundante, favorecen la aparición de especies como $H$. turcicus, $T$. mauritanica, y $P$. hispanica, bastante ligada a medios rupícolas y soleados (MELLADO, 1980). Así como algunos ofidios: $N$. maura, favorecida por las construcciones para retención de agua, y $M$. monspessulanus, que, aunque ha sido encontrada principalmente en estos medios, no se debe a su especial predilección, sino por ser donde más ejemplares muertos se detectan, debido al paso de vehículos o a la simple presencia humana.

Se comprueba que la riqueza de reptiles (medida tanto en número de especies como en diversidad), está correlacionada con la riqueza vegetal, ajustándose bien a un modesto lineal, no dependiendo de la altura y grado de cobertura de la vegetación (Tabla IV, Figura 7). Para esta comparación, no han sido considerados los biotopos antropógenos y cultivos por no ser cuantificables en ellos la riqueza vegetal.

La expresión que encontramos más interesante, desde el punto de vista práctico para su utilización en trabajo de campo, es la que nos relaciona el número de especies animales con el número de especies vegetales, que un simple conteo de estas últimas, permite hacer una estimación rápida del número de especies de reptiles que cabe esperar en ese medio. Según la expresión:

$$
\text { N.S.A. }=0.43 \text { N.S.V. }-2.87
$$

(N.S.A.: número de especies animales; N.S.V.: número de especies vegetales), sería posible encontrar las trece especies detectadas en un mismo biotipo compuesto de 37 especies vegetales distintas. La expresión citada, no pretende ser más que una aproximación teórica, ya que para una estimación precisa habría que considerar otras muchas características y variables del biotopo. 


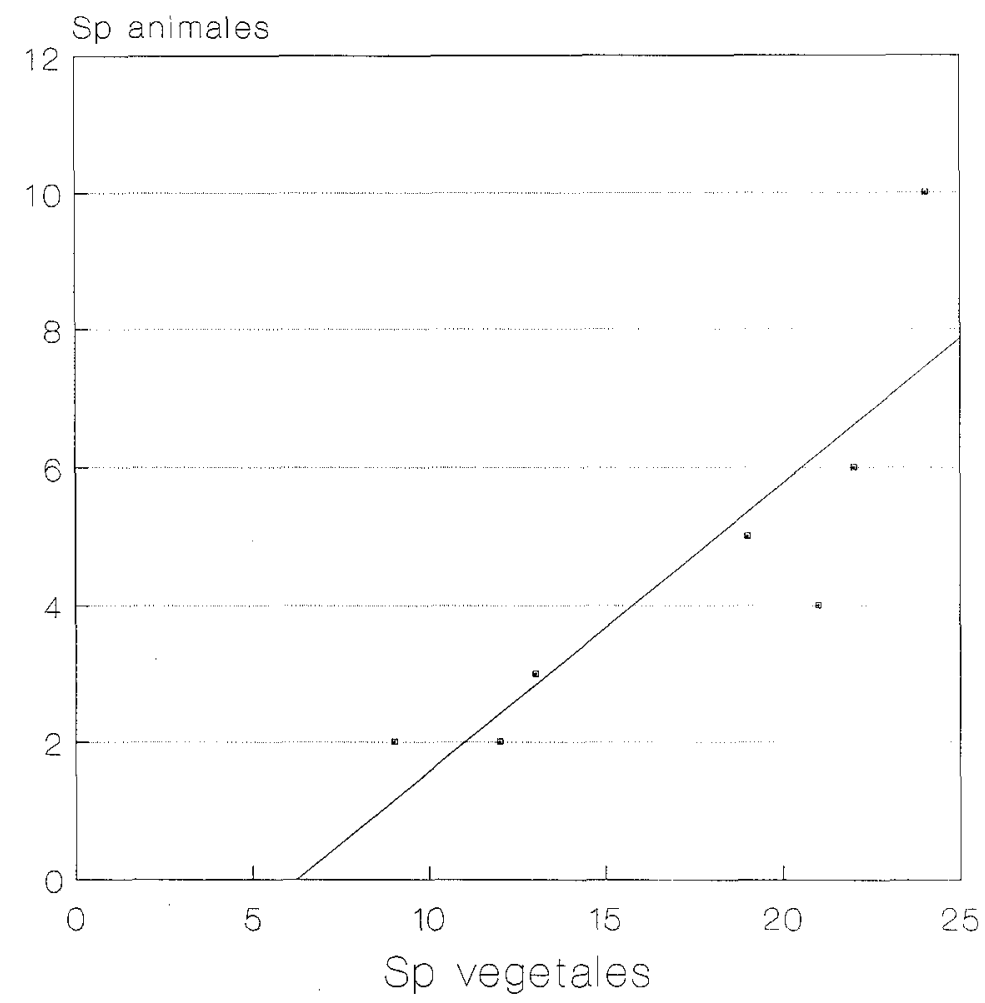

Figura 7.- Ajuste lineal entre el $n .{ }^{\circ}$ de especies de reptiles y el $n .{ }^{\circ}$ de especies vegetales de los biotopos naturales y terrenos improductivos.

\subsection{Relaciones específicas entre saurios}

Debido a la escasez de citas de Ofidios y Amphisbaénidos, hemos realizado el estudio de las relaciones entre especies, tan sólo para los Lacértidos y Gekkónidos, ya que han sido los taxones más abundantes en la comarca, ofreciéndonos datos suficientes para extraer conclusiones en este sentido.

Para establecer relaciones se ha medido, mediante el porcentaje de ocupación en los distintos biotopos, la capacidad colonizadora de cada especie, y con estos datos se ha calculado el grado de solapamiento que existe entre ellas.

En la Tabla V, se puede comprobar que el índice de solapamiento mayor aparece entre $H$. turcicus y $T$. mauritanica, y con un valor similar, entre L. lepida y Ps. hispanicus. El primer caso es debido a que estas especies aparecen juntas en la mayoría de biotopos que habitan, siendo éstos, como ya hemos visto, las estructuras antropógenas. En el segundo caso, se debe a que estas dos especies comparten la capacidad de adaptarse a terrenos 


\section{TABLA V}

\begin{tabular}{lccccccc}
\hline & HET & TAM & ACE & LAL & POH & PSA & PSH \\
\hline HET & 1.00 & 0.91 & 0.00 & 0.15 & 0.54 & 0.15 & 0.06 \\
TAM & & 1.00 & 0.00 & 0.49 & 0.51 & 0.28 & 0.43 \\
ACE & & & 1.0 & 0.00 & 0.00 & 0.29 & 0.00 \\
LAL & & & & 1.00 & 0.49 & 0.70 & 0.90 \\
POH & & & & & 1.00 & 0.75 & 0.13 \\
PSA & & & & & & 1.00 & 0.42 \\
PSH & & & & & & & 1.00 \\
\hline
\end{tabular}

Tabla V.- Índice de solapamiento del nicho espacial definido por los biotopos (Fam: Lacertidae y Gekkonidae).

muy degradados como son los improductivos, aunque esta capacidad es superior en PS. hispanicus (ver ap. 4.1.).

También existe amplio solapamiento entre $P$. algirus, $L$. lepida y $P$. hispanica, resultando similar al obtenido por PÉREZ-MELLADO (1982). En menor grado, también se obtiene un ligero solapamiento entre $P$. hispanica, $H$. turcicus y $T$. mauritanica, debido a que comparten preferencias rupícolas y fisurícolas (MELLADO et al., 1975). Dentro de la comunidad aparece $A$. erythrurus como la más especializada en la utilización del espacio, (ver ap. 4.1.), por lo que no presenta solapamiento con ninguna especie.

Una representación gráfica de estas relaciones se observa en el dendrograma obtenido en base a las abundancias específicas encontradas en cada biotopo (Figura 8), donde aparecen unidas nuevamente $H$. turcicus y $T$.

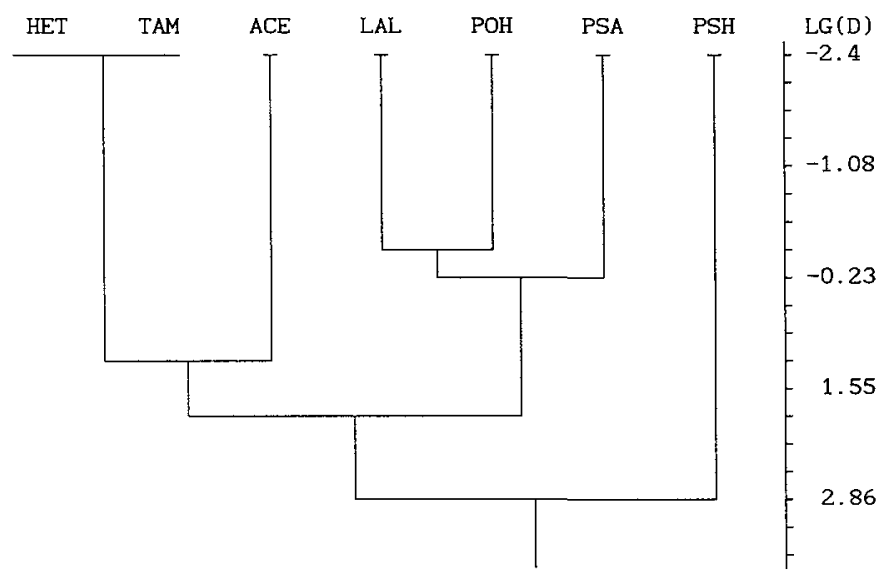

Figura 8.- Dendrograma de Afinidad obtenido mediante Análisis de Agrupamiento de las Familias Lacertidae y Gekkonidae en relación con los biotopos prospectados. 
mauritanica, y éstas a su vez, aunque alejadas, se sitúan junto a $A$. erythrurus, debido a que comparten la característica de tener preferencias de habitat específico. Por otro lado aparece un segundo grupo donde se encuentran bastante próximas nuevamente las especies $L$. lepida, $P$. hispanica y $P$ s. algirus, ya que comparten algunas preferencias de habitats, aunque de ellas es $P$. algirus la que mejor coloniza la vegetación natural.

Por último, se segrega Ps. hispanicus del resto, ya que se muestra como la especie más abundante de la comunidad, y la más favorecida en áreas degradadas, siendo el saurio que mejor coloniza estos biotopos.

\section{Distribución altitudinal}

Para el estudio de esta distribución se ha dividido la comarca en siete intervalos altitudinales de $100 \mathrm{~m}$. cada uno a partir del nivel del mar. En el último se han considerado todas las alturas mayores de 600 m.s.n.m., ya que son muy pocas las cuadrículas por encima de esta cota.

En este apartado se han utilizado tanto los datos obtenidos de la prospección de campo, como los preexistentes en el archivo faunístico, ya que, mediante los mapas U.T.M. a escala 1:50000, nos ha sido posible asignar la altitud a cada una de estas últimas citas.

En la Figura 9.a se puede observar el índice de abundancia calculado para cada intervalo, encontrando un máximo entre las cotas de 300 y 400 m.s.n.m.

La riqueza total obtenida (Figura 9b), es decir, el número máximo de especies detectadas en cada intervalo, sigue una tendencia a la disminución desde la clase más baja, con las trece especies representadas, hasta el intervalo número III ( 200 - 300 m.s.n.m.), a partir del cual sufre un pequeño aumento y una estabilización progresiva.

El estudio de la riqueza es una medida aproximada de la diversidad. Así, se puede observar en la Tabla VI que la diversidad de Shannon-Weaver es máxima en los dos primeros intervalos altitudinales. Sin embargo, para los intervalos III y VII, aunque con bajo índice de abundancia, se obtiene una equitabilidad bastante alta, debido a la escasez de especies que hemos encontrado en estos intervalos.

El estudio de la distribución altitudinal en la comarca no resulta suficientemente significativo como para caracterizar la comunidad, ya que la diferencia de cotas que se puede encontrar va desde el nivel del mar hasta 1.200 m.s.n.m. en el Cabeço d'Or, y se puede encuadrar en su totalidad como pisos bajos para la distribución de reptiles.

Tan sólo las dos especies de salamanquesas $(H$. turcicus y $T$. mauritanica), y $A$. erythrurus, se ven algo afectadas por la altitud, en parte por ser especies termófilas. De hecho $H$. turcicus presenta una distribución costera en la Península (SALVADOR, 1985), y T. mauritanica en la cara norte de la Sierra de Gredos no supera los 1.000 m.s.m. (LIZANA et al., 1987). Asimismo, las tres especies coindicen, además de su tendencia termófila, en su preferencia por habitat específicos. Las dos primeras se muestran cla- 


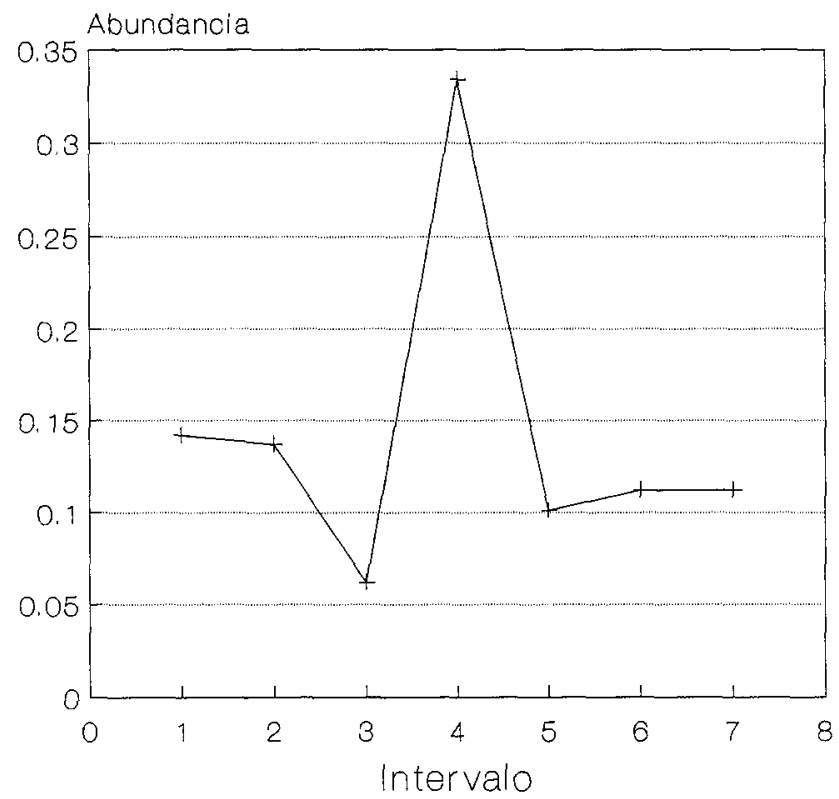

A

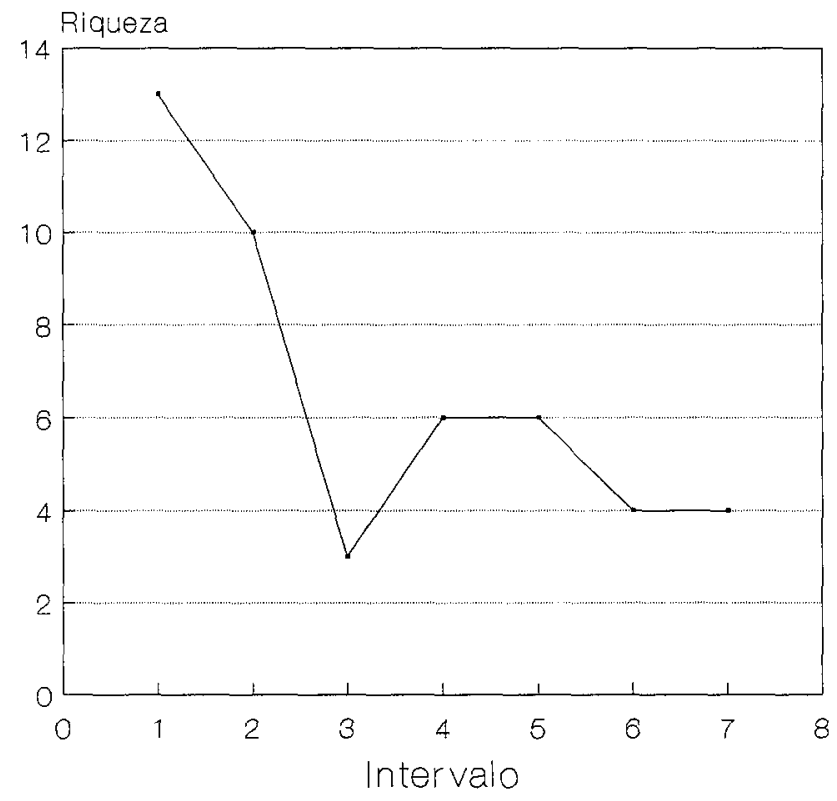

B

Figura 9.- Abundancia (A) y Riqueza (B) de reptiles de cada intervalo altitudinal. 


\begin{tabular}{lcclcccc}
\hline INT. ALT & I & II & III & IV & V & VI & VII \\
\hline S & 13 & 10 & 3 & 6 & 6 & 4 & 4 \\
n & 146 & 56 & 5 & 13 & 27 & 9 & 6 \\
H' & 2.03 & 1.94 & 1.05 & 1.48 & 1.44 & 1.00 & 1.33 \\
E & 0.79 & 0.84 & 0.96 & 0.83 & 0.80 & 0.72 & 0.96 \\
\hline
\end{tabular}

Tabla VI.- Diversidad de Shannon-Weaver $\left(\mathrm{H}^{\prime}\right)$ y Equitabilidad de Pielou (E) de la comunidad de reptiles, para cada intervalo altitudinal de $100 \mathrm{~m}$. desde el nivel del mar ( $\mathrm{S}=\mathrm{n}^{\circ}$ de especies, $\mathrm{n}=\mathrm{n}^{\circ}$ de individuos).

ramente antropófilas (ver ap. 4.1.), y los habitat antropizados son dominantes en las cotas más bajas de la comarca, destacando en los términos municipales de San Juan, San Vicente del Raspeig y Muchamiel (SEVA y PINA, 1981). La tercera, $\boldsymbol{A}$. erythrurus, muestra una preferencia de habitat por el arenal costero de la comarca (ver ap. 4.1.), y por lo tanto sólo frecuenta las cotas más bajas.

Una especie que también se muestra por zonas bajas y medias es $B$. cinereus debido a que está ligada a campos de cultivo y sus cercanías (DICENTA et al., 1986).

El resto de las especies tiene un intervalo altitudinal más amplio (PLEGUEZUELOS, 1986) que el representado en la comarca, y su ausencia en algunas cotas, como en el caso de los Ofidios, se debe probablemente más a su baja densidad que a su segregación.

\section{CONCLUSIONES}

1. Las abundancias de las familias Colubridae y Amphisbaenidae en la comarca son, cuando menos, un orden de magnitud inferior a las de Lacertidae y Gekkonidae. Para conseguir resultados similares a los encontrados en éstas dos últimas familias, y suficientes para los análisis estadísticos, habría que incrementar proporcionalmente la intensidad de prospección.

2. El valor máximo de abundancia por especies se obtiene en $P$. hispanicus, y el biotopo con mayor índice de abundancia es el constituido por los terrenos improductivos, debido a a la fácil colonización de éstos por parte de dicha especie, principalmente, seguida de L. lepida, que también está dentro del rango de las especies más abundantes. Por tanto, la densidad de ambas sobre las demás especies, nos sirve como característica indicadora del estado de degradación y fraccionamiento de la vegetación natural.

3. Dentro de las zonas con influencia humana, es el medio constituido por construcciones rústicas o puntuales, el que menos modificaciones provoca en la comunidad de reptiles. Las especies que aumentan sus efectivos cuando un medio está ocupado principalmente por estas características son $H$. turcicus y $T$. mauritanica. 
4. Dentro de la vegetación natural, la mayor riqueza de reptiles se obtiene en el tomillar, que es el biotopo más abundante de la vegetación natural, a pesar de su fragmentación, ofreciendo unos resultados óptimos de abundancia y diversidad. Se comprueba que la riqueza animal depende de la riqueza vegetal del medio; por tanto podemos decir, que el desarrollo óptimo de la comunidad herpetológica depende de la integridad del medio y la conservación de especies vegetales en los medios naturales en general.

En definitiva, podemos decir que los resultados obtenidos en relación con la abundancia, diversidad, y distribución de los reptiles en la comarca, se debe a una progresiva pérdida de diversidad estructural, como consecuencia del gran impacto humano que existe en el territorio, lo que ha provocado una creciente degradación y fragmentación de la vegetación natural, quedando ésta acantonada como pequeñas islas, cada vez más difíciles de conservar, y deteriorando las poblaciones de reptiles. Este mismo problema se observa en otros puntos de la península (véase SANTOS y TELLERIA, 1989), y su solución depende de la futura gestión que se haga sobre el uso del suelo, tanto desde el punto de vista urbanístico y agrícola, como conservacionista.

\section{BIBLIOGRAFÍA}

ALCARAZ, F. \& M. PEINADO, 1987. El Sudeste Ibérico Semiárido, en La Vegetación de España, M. Peinado y S. Rivas - Martínez (eds.). Serv. Publ. Univ. Alcalá de Henares: 259-281.

ARNOLD, E. N. \& J. A. BURTON, 1978. Guía de Campo de los Anfibios y Reptiles de España y Europa. Omega. 257 pp.

AUERNHEIMER C., J. A. PINA \& A. I. ECHEVARRIA, 1981. Geología Ambiental de la Comarca de Alicante, en El Medio Físico de la Comarca de Alicante. Excmo. Ayto. de Alicante: 51-54.

- \& A. ESCARRE, 1981. El Medio Físico de la Comarca de Alicante. Excmo. Ayto. de Alicante.

— \& J. A. PINA, 1983. Problemática Geoambiental del Campo de Alicante. Hombre y Medio Natural en Alicante. Secr. Publ. Univ. Alicante: 15-31.

BARTOLOMÉ, F., 1985. Aproximación al clima en la Isla Plana o Nueva Tabarca (Alicante), en La Reserva Marina de la Isla Plana o Nueva Tabarca (Alicante), A. A. Ramos (ed.) Excmo. Ayto. Alicante y Secr. Publ. Univ. Alicante: 37-49.

CANO, C., 1984. La Comunidad de Lacertidos (Lacertidae, Squamata) de un encinar continental. Tesina de Licenciatura. Univ. Compl. de Madrid.

CAUGHLEY, G., 1977. Analysis of Vertebrate Populations. Wiley and Sons. Londres.

DICENTA, F., V. HERNÁNDEZ \& F. ROBLEDANO, 1986. Contribución al Atlas Herpetológico de la Región de Murcia. I Congreso Nacional de Herpetología. Benicássim. 30 pp. 
ESCARRE, A. \& J. R. VERICAD, 1981. Fauna Alicantina: I. Saurios y Ofidios. Exma. Diputación de Alicante. $101 \mathrm{pp}$.

-, J. MARTIN, E. SEVA, J. BELlot, M. J. LLEDO \& C. PINA, 1981. Análisis de la cubierta vegetal en la Comarca de Alicante, en El Medio Físico de la Comarca de Alicante. Excmo. Ayto. de Alicante. 87-159.

- J. MARTÍN, E. SEVA, J. BELLOT, M. J. LLEDO \& C. PINA \& E. FERRANDIS, 1983. Tipificación y características de la cubierta vegetal del Camp d'Alacant. Hombre y Medio Natural en Alicante. Secr. Publ. Univ. Alicante: 15-31.

JAMES, F. C. \& S. RATHBUN, 1981. Rarefaction, relative abundance and diversity of avian communities. Auk, 98: 785-800.

LIZANA M., J. M. CIUDAD \& V. PÉREZ-MELLADO, 1987. Distribución Altitudinal de la herpetofauna en el Macizo Central de la Sierra de Gredos. II Congreso Nacional de Herpetología. Salamanca. $10 \mathrm{pp}$.

MACARTHUR, R. \& R. LEVINS, 1967. The limiting similarity, convergence and divergence of coexisting species. American Naturalist, 101: 377-385.

MARGALEF, F., 1986. Ecología. Omega. $951 \mathrm{pp.}$

MAY, R. M. 1974. On the teory of niche overlap. Theor. Pop. Biol., 5: 297-332.

MELLADO, J., 1980. Utilización del espacio en una comunidad de lacértidos del matorral mediterráneo en la Reserva Biológica de Doñana. Doñana Acta Vertebrata, 7 (1): 41-59.

- F. AMORES, F. F. PARREÑO \& F. HIRALDO, 1975. The structure of a Mediterranean lizard community. Doñana Acta Vertebrata, 2 (2): 145-160.

PÉREZ-MELLADO, V. 1982. Estructura de una taxocenosis de Lacertidac (Sauria, Reptilia) del Sistema Central. Mediterránea, 6: 39-64.

- 1983. La herpetofauna de Salamanca: un análisis biogeográfico y ecológico. Salamanca. 9: $10-78$.

- 1989. Ecología de los Anfibios y Reptiles. I Curso de Herpetología y Toxicología. Academia de Ciencias Veterinarias de Cataluña, 35 pp.

PLEGUEZUELOS, J. M., 1986. Distribución altitudinal de los Repties en las Sierras Béticas orientales. Revista Española de Herpetología, 1: 63-84.

RIGUAL, A., 1972. Flora y Vegetación de la Provincia de Alicante, Instituto de Estudios Alicantinos. Diputación de Âlicante. 403 pp.

RIVAS-MARTÍNEZ, S, 1987. Nociones sobre Fitosociología, Biogeografía y Bioclimatología, en La Vegetación de España, M. Peinado y S. Rivas-Martínez (eds.) Serv. Publ. Univ. Alcalá de Henares: 19-47.

SAlvadOR, A., 1985. Guía de Campo de los Anfibios y Repties de la Península Ibérica, Islas Balcares y Canarias. S. García (ed.) León. 212 pp. y mapas.

SANTOS, T. \& J. L. TELLERIA, 1989. Preferencias de habitat y perspectivas de conservación en una comunidad de Lacértidos en medios cerealistas del centro de España. Revista Española de Herpetología, 3 (2): 259-273.

SCHERRER, B., 1983. Techniques de sondage en écologie, en Stratégies d'echantillonnage en écologie. S. Frontier (ed.) Masson: 63-162. Paris.

SEVA, E., 1982. Taxocenosis de Lacertidos en un arenal costero alicantino. Tesis Doctoral. Secr. Publ. Univ. Alicante. 317 pp.

- \& A. ESCARRE, 1976. El Eslizón Ibérico (Chalcides bedriagai) en el medio insular de Nueva Tabarca (Provincia de Alicante). Mediterránea, 1: 61-115.

- \& J. A. PINA, 1981. Estructuras Antropógeas en la comarca de Alicante. Banco de Datos, en El Medio Físico de la Comarca de Alicante. Excmo. Ayto. de Alicante: 159-195.

- E. FERRANDIS \& A. ESCARRE, 1982. La selección de habitat del arenal costero alicantino por Acanthodactylus erythrurus (Sauria, Lacertidae). Un proceso markoviano para la identificación de sus tendencias. Mediterránea, 6: 5-14.

- \& A. ESCARRE, 1983. Estrategias en el reparto de recursos en cuatro especies de saurios del arenal alicantino. En: Hombre y Medio Natural en Alicante. Secr. Publ. Univ. Alicante: 123-131.

TELlERIA, J. L., 1986. Manual para el Censo de Vertebrados Terrestres. Raíces 278 pp. Madrid. 